\title{
Sequential projection pursuit for optimized vibration-based damage detection in an experimental wind turbine blade
}

\author{
Simon Hoell ${ }^{1}$ \& Piotr Omenzetter ${ }^{2}$ \\ ${ }^{1}$ Institute of Structural Mechanics, Bauhaus-University Weimar, 99423 Weimar, \\ Germany \\ 2 The LRF Centre for Safety and Reliability Engineering, The University of \\ Aberdeen, Aberdeen AB24 3UE, UK \\ E-mail: piotr.omenzetter@abdn.ac.uk \\ August 2017
}

\begin{abstract}
To advance the concept of smart structures in large systems, such as wind turbines, it is desirable to be able to detect structural damage early while using minimal instrumentation. Data-driven vibration-based damage detection methods can be competitive in that respect because global vibrational responses encompass the entire structure. Multivariate damage sensitive features (DSFs) extracted from acceleration responses enable to detect changes in a structure via statistical methods. However, even though such DSFs contain information about the structural state, they may not be optimized for the damage detection task. This paper addresses the shortcoming by exploring a DSF projection technique specialized for statistical structural damage detection. High dimensional initial DSFs are projected onto a low-dimensional space for improved damage detection performance and simultaneous computational burden reduction. The technique is based on sequential projection pursuit where the projection vectors are optimized one by one using an advanced evolutionary strategy. The approach is applied to laboratory experiments with a small-scale wind turbine blade under wind-like excitations. Autocorrelation function coefficients calculated from acceleration signals are employed as DSFs. The optimal numbers of projection vectors are identified with the help of a fast forward selection procedure. To benchmark the proposed method, selections of original DSFs as well as principal component analysis scores from these features are additionally investigated. The optimized DSFs are tested for damage detection on previously unseen data from the healthy state and a wide range of damage scenarios. It is demonstrated that using selected subsets of the initial and transformed DSFs improves damage detectability compared to the full set of features. Furthermore, superior results can be achieved by projecting autocorrelation coefficients onto just a single optimized projection vector.
\end{abstract}

Keywords: autocorrelation function, evolutionary algorithm, optimal feature projection, sequential projection pursuit, structural health monitoring, vibrationbased damage detection, wind turbines

Submitted to: Smart Mater. Struct. 


\section{Introduction}

The evolution of many conventional aerospace, mechanical, civil and other structural system towards smart structures is inevitable in the current competitive economic environment and increasing public interest in eco-friendliness, safety and reliability of these systems. This is especially true for novel energy systems, such as wind turbines (WTs), where relentless demands for higher energy production capacities lead to growing sizes of WTs and erections in remote areas with higher and more predictable average wind speeds. However, expected revenues and energy production targets are adversely affected by increasing operations and maintenance expenditures, which can make up to $20 \%$ of the total energy production costs [1]. To address the challenge by turning WTs into smart systems, the development of efficient structural damage detection (SDD) technologies is essential because they play a key role in quantitative assessment of the current structural state using the information and knowledge extracted from measured physical quantities [2].

Various techniques based on different physical principles have been proposed in the past for SDD, including acoustic emission [3], fibre optic strain measurements [4] and active vibration techniques using ultrasonic waves [5]. However, the local nature of these methods requires dense sensor arrays, which adds to instrumentation and data analysis costs. Contrarily, passive vibration-based methods utilize vibrational responses resulting from ambient and/or operational sources, such as wind and rotor rotation in the case of WTs. These responses encompass the entire structure due to low damping and long wavelengths. Furthermore, they are affected by changes in stiffness, energy dissipation mechanisms and mass, which are typical results of structural damage. Thus, SDD can be performed using modest amount of instrumentation.

Damage sensitive features (DSFs) are extracted from vibration signals to reduce data volumes and to improve damage detectability. These DSFs are usually multivariate and include modal parameters [6], parametric time series model coefficients [7-10], and non-parametric time series representations in the frequency domain $[11,12]$, time-frequency domain [13] or time domain. For the latter, amplitudes of cross-correlations [14] and their coefficients [15] or cross-correlations [16] and autocorrelations [17] at the zero lag were all proposed for SDD. These DSFs are attractive due to their sensitivity to damage and low computational requirements their estimation entails. Therefore, autocorrelation coefficients (ACCs) are selected as DSFs in the present study.

However, irrespective of the multivariate DSF type adopted, high feature dimensions and/or inclusion of components which are noisy or insensitive to damage may cause problems when resources for computation and transfer of data are limited, e.g. in wireless sensor applications [18], or blunt the performance of a SDD algorithm due to the curse of dimensionality. The latter manifests as shortening of distances to high-dimensional outliers, and it becomes more difficult to distinguish them in noisy data [19]. Hence, the present research aims at identifying projections and selections of DSFs which will allow improving damage detectability and, at the same time, reducing the feature dimensions. Forward schemes for selecting features, where components are iteratively added to a DSF vector, were studied by Park et al. [20] and Zugasti et al. [21]. A more general approach was studied by Worden et al. [22] using a genetic algorithm for finding optimal feature subsets. Although selecting initial DSFs can improve results, discarding all the information about the structural state contained in the ignored components may not lead to optimal SDD results. Appropriate transformations of the initial features can be used to retain the information in a smaller numbers of highly sensitive transformed DSFs while undesired effects from noise or environmental and operational changes can be reduced. Principal component analysis (PCA) aims at identifying orthogonal bases which maximize the variation in a dataset in the first few dimensions [23]. This property can facilitate both aims, i.e. dimensionality reduction and removal of adverse effects, by making assumptions about sources of DSF variations. The application of PCA for feature extraction received considerable attention in the past [24-27]. However, even though these techniques can improve SDD performance, they were not originally developed for this task. Thus, the characteristics of DSFs in different structural states and properties of the SDD algorithm can not be taken into account. Suboptimal results are often a consequence.

The present study builds on and extends the authors' previous work presented in [8] and [28], where optimal selections of the initial and PCA-transformed time series-based DSFs were studied, respectively. The previous research demonstrated, via tracktable conceptual examples, and validated, through extensive numerical and experimental studies, that, depending on the correlation between the initial DSF components and their sensitivity to damage, different selections and projections of DSFs can be optimal for SDD. This paper introduces a novel and improved method for SDD. The new method employs the sequential projection pursuit (SPP) concept in combination with an advanced evolutionary optimization algorithm for obtaining the optimal DSF projection vectors. This approach offers more flexibility and further improvements in damage detectability, because the 
vector directions are unrestricted and can be tailored to all the available data and anticipated damage states, in contrast to the previously considered selections from the initial or PCA-transformed DSFs that were determined by the healthy state data only. Additionally, a gradient-free optimization algorithm is adopted that allows incorporating complex objective functions specialised for SDD problems at hand. SPP enables to reduce the computational burden, because its projection vectors can be estimated iteratively [29].

The method was applied to laboratory experiments with a small-scale wind turbine blade (WTB) made of a glass-fibre reinforced composite material. Non-contact wind-like excitation was applied using a household pedestal fan, and several scenarios combining different damage locations and extents were simulated by attaching small masses. (Note, we refer herein to the attached masses as 'damage' although real damage would rather take form of stiffness reduction. Using masses, however, allowed to study a much wider range of so-understood damages nondestructively compared to what could have been practical had we introduced irreversible cuts or similar.) Initial DSFs were defined as the ACCs estimated from vibration responses. Statistical hypothesis testing was employed for SDD due to its computational efficiency and conceptual clarity.

The paper is structured as follows. First, the working of the proposed SDD method is explained and the underlying theory presented. Second, an analytical illustrative example is provided to highlight the main features and benefits of the proposed SDD method. Third, an application of the SPP-based SDD method to the WTB experiments is discussed and the improved performance is demonstrated in comparison to using the initial and PCA-transformed DSFs. Finally, conclusions are drawn and directions for future work presented.

\section{Methodology and theory}

The framework of the proposed methodology for advanced vibration-based SDD consists of a training and an operational phase. The training phase uses vibration response data (accelerations) from different structural states, $x_{s}[t]$, where index $s$ refers to a given state and $t$ is the time instant, respectively. They can be divided into $n$ segments of common sample numbers, $x_{s, i}[t](i=1,2, \ldots, n)$. Standard, zero-mean, normally distributed time series segments, $z_{s, i}[t]$, can be obtained from $x_{s, i}[t]$ by removing estimated means, $\hat{\mu}_{x_{s, i}}$, and dividing by estimated standard deviations, $\hat{\sigma}_{x_{s, i}}$, of each segment. Then, initial DSF vectors, $\mathbf{v}_{s, i}$, can be constructed. Consecutive ACCs are used in our research because they can be efficiently estimated and are sensitive to damage $[30,31]$. A training database comprising DSF matrices from different structural conditions, $\mathbf{V}_{s}=\left[\begin{array}{llll}\mathbf{v}_{s, 1} & \mathbf{v}_{s, 2} & \ldots & \mathbf{v}_{s, n}\end{array}\right]$, is created. Then, the actual training begins with sequential identification of a projection matrix, $\mathbf{T}$, optimized with respect to a predefined objective function, $J(\mathbf{T})$. The objective function is defined as the minimum of a relative statistical distance between the healthy and selected damage states. Finally, the operational stage SDD is performed with the help of statistical hypothesis testing, where optimally transformed DSFs from the reference healthy state and the current state are used for calculating statistical distances. The basic details of the theories underlying the procedural steps are given in the following sections. First, the initial DSFs are defined as ACCs. Second, PCA is briefly explained because it is herein employed as a benchmark for comparing the performance of SPP. In the next section, SPP is introduced, including a discussion of the projection optimization algorithm. Then, statistical hypothesis testing is described. Finally, the fast forward feature selection algorithm and the objective function are presented.

\subsection{Autocorrelation-based damage sensitive features}

The ACCs are used herein as the initial DSFs. Unbiased estimates of ACCs, $r_{s, i}[\tau]$, at time lag $\tau$ for a discrete zero-mean time series segment, $z_{s, i}[t]$, with $n_{\text {samp }}$ samples can be calculated as [30]:

$$
r_{s, i}[\tau]=\frac{1}{n_{\text {samp }}-\tau} \sum_{k=1}^{n_{\text {samp }}-\tau} z_{s, i}[k] z_{s, i}[k+\tau]
$$

In order to employ statistical tests for identifying a structural state, an appropriate probability distribution function needs to be selected for the DSF. Since ACCs are bounded between -1 and +1 , theoretical expressions about their distributions are not obvious. Therefore, Fisher's z-transformation is applied to obtain the approximately normally distributed $z$-scores, $r_{s, i}^{z}[\tau]$, from the ACCs [32]:

$$
r_{s, i}^{z}[\tau]=\tanh ^{-1}\left(r_{s, i}[\tau]\right)
$$

Multivariate DSF vectors, $\mathbf{v}_{s, i}$, can be defined taking the $z$-scores of the first $m$ ACCs as:

$$
\mathbf{v}_{s, i}=\left[\begin{array}{llll}
r_{s, i}^{z}[1] & r_{s, i}^{z}[2] & \cdots & r_{s, i}^{z}[m]
\end{array}\right]^{T}
$$

where superscript $T$ denotes transpose. The DSF vectors, $\mathbf{v}_{s, i}$, follow then a multivariate Gaussian distribution, $\mathcal{N}\left(\boldsymbol{\mu}_{s}, \boldsymbol{\Sigma}_{s}\right)$, with mean vector $\boldsymbol{\mu}_{s}$ and variance-covariance matrix $\boldsymbol{\Sigma}_{s}$.

\subsection{Principal component analysis}

Principal component projections of DSFs are used in this study to benchmark the performance of SPP. 
Using DSF vectors from the healthy state, $\mathbf{v}_{h, i}$, from $n$ time series segments and removing the estimated mean, $\hat{\boldsymbol{\mu}}_{h}$, a DSF matrix can constructed as:

$$
\mathbf{V}_{h}=\left[\begin{array}{llll}
\mathbf{v}_{h, 1}-\hat{\boldsymbol{\mu}}_{h} & \mathbf{v}_{h, 2}-\hat{\boldsymbol{\mu}}_{h} & \cdots & \mathbf{v}_{h, n}-\hat{\boldsymbol{\mu}}_{h}
\end{array}\right]
$$

(It should be noted that in this study only the healthy state DSF samples are used for PCA.) PCA identifies a linear transformation, $\mathbf{V}_{P C}=\mathbf{T}_{P C}^{T} \mathbf{V}_{h}$, such that the resulting features/scores, $\mathbf{V}_{P C}$, are linearly independent and their variances are maximized in the first few dimensions. The transformation matrix, $\mathbf{T}_{P C}$, can be obtained by singular value decomposition of the DSF variance-covariance matrix, $\boldsymbol{\Sigma}_{h}$ :

$$
\boldsymbol{\Sigma}_{h}=\mathbf{T}_{P C} \boldsymbol{\Lambda} \mathbf{T}_{P C}^{T}
$$

The diagonal matrix $\boldsymbol{\Lambda}$ contains the eigenvalues of $\boldsymbol{\Sigma}_{h}$ arranged in the descending order by the variance of the corresponding PCA scores. The principal components (PCs) are the normalized eigenvectors stored as column vectors in $\mathbf{T}_{P C}$. The score matrix, $\mathbf{V}_{P C}$, contains the PCA scores as its columns.

\subsection{Sequential projection pursuit}

Projection pursuit is a technique initially developed for exploratory data analysis [33]. It aims at identifying a projection matrix, $\mathbf{T}_{P P}$, containing $p m$ dimensional projection vectors, where $p \ll m$. This matrix is established by optimization of a problemspecific objective function, $J(\mathbf{T})$, which measures the 'interestingness' of the resulting projection directions. ('Interestingness' is context-dependent and refers to how well the particular projections explain the phenomenon being studied.) The optimization problem can be formulated as follows:

$$
\begin{aligned}
\mathbf{T}_{P P}= & \underset{\mathbf{T}}{\arg \max }\{J(\mathbf{T})\} \\
\text { subject to } & \mathbf{T T}^{T}=\mathbf{I}
\end{aligned}
$$

where the normality is imposed to avoid information redundancies between individual projections and orthogonality to ensure solution uniqueness. However, practically useful or necessary objective functions are often multi-modal with many local minima or functions whose derivatives cannot be analytically derived, which renders the application of gradient-based optimization techniques difficult. On the other hand, gradientfree optimization strategies may suffer from the large number of variables required.

The SPP tries to overcome those limitations by splitting the problem of finding the whole $(p \times m)$ dimensional matrix into finding the $p m$-dimensional projection vectors one by one [29]. It was inspired by the sequential formulation of PCA, where the $\mathrm{PCs}$ are also identified one-by-one by maximizing their variances. An appropriate number of projection vectors is generally not known a priori. The sequential estimation of the projection vectors in SPP is beneficial because more vectors can easily be added to the previous ones if initial results are unsatisfactory. In the original SPP approach by Guo et al. [29], the previously projected data was removed between the sequential steps. However, in SDD applications where objective functions are affected by the interrelations between projected DSFs from different damage states, the removal may lead an overall suboptimal performance. Therefore, we propose instead the objective function at each sequential step be evaluated using a fixed projection matrix established in the previous steps while varying only the new projection vector such that the orthonormality constraints are fulfilled.

The reduced number of variables allows employing gradient-free global optimization strategies. Espezua et al. [34] studied the application of genetic algorithms for SPP. However, in the area of evolutionary global optimizers [35], one of the recent promising developments is the powerful covariance matrix adaptation-evolutionary strategy by Hansen and Ostermeier [36]. The algorithm generates offspring individuals in each generation by sampling from a multivariate Gaussian probability distribution whose statistical parameters are adapted based on the evaluation of previous generations. The mean vector moves towards the optimum, while the adaptation of the variance-covariance matrix controls the mutation rate in order to favour generation of potentially more successful candidates.

\subsection{Statistical hypothesis testing}

For deciding systematically and rigorously whether a structure is healthy or damaged, statistical hypothesis testing is an efficient tool, which additionally enables to compare the performances of different DSF types. A statistical hypothesis test for the mean of a multivariate DSF from the current state, $\boldsymbol{\mu}_{c}$, against the healthy state mean, $\boldsymbol{\mu}_{h}$, transformed using a projection matrix, $\mathbf{T}$, can be defined as follows:

$$
\begin{array}{lll}
H_{0}: & \mathbf{T}^{T} \boldsymbol{\mu}_{c}=\mathbf{T}^{T} \boldsymbol{\mu}_{h} & \text { (healthy) } \\
H_{1}: & \mathbf{T}^{T} \boldsymbol{\mu}_{c} \neq \mathbf{T}^{T} \boldsymbol{\mu}_{h} & \text { (damaged) }
\end{array}
$$

where the null hypothesis, $H_{0}$, represents the healthy state, and the alternative hypothesis, $H_{1}$, corresponds to the damage state. Since the true statistical properties are generally unknown and have to be estimated from available data, the $T^{2}$ statistic is herein employed [37]:

$$
T^{2}(\mathbf{T}, a, b)=\frac{n_{a} n_{b}}{n_{a}+n_{b}}\left(\hat{\boldsymbol{\mu}}_{a}-\hat{\boldsymbol{\mu}}_{b}\right)^{T} \mathbf{T} \hat{\boldsymbol{\Sigma}}_{p l}^{-1} \mathbf{T}^{T}\left(\hat{\boldsymbol{\mu}}_{a}-\hat{\boldsymbol{\mu}}_{b}\right)
$$


where $n_{a}$ and $n_{b}$ are the sample numbers of two different states, and the hat denotes estimated quantities. The estimated pooled variance-covariance matrix, $\hat{\boldsymbol{\Sigma}}_{p l}$, is defined as:

$$
\hat{\boldsymbol{\Sigma}}_{p l}=\frac{\left(n_{a}-1\right) \mathbf{T}^{T} \hat{\boldsymbol{\Sigma}}_{a} \mathbf{T}+\left(n_{b}-1\right) \mathbf{T}^{T} \hat{\boldsymbol{\Sigma}}_{b} \mathbf{T}}{n_{a}+n_{b}-2}
$$

Under the assumption of multivariate Gaussian distributed DSFs, $\mathbf{T}^{T} \mathbf{v} \sim \mathcal{N}\left(\mathbf{T}^{T} \boldsymbol{\mu}, \mathbf{T}^{T} \boldsymbol{\Sigma} \mathbf{T}\right)$, the $T^{2}$ statistic follows Hotelling's probability distribution, $\mathcal{T}_{r, n_{a}+n_{b}-2}^{2}$, with $r$ and $n_{a}+n_{b}-2$ degrees of freedom (DOFs), where $r$ is the rank of $\mathbf{T}$. The hypotheses can be tested using the inverse cumulative Hotelling's probability distribution function $F_{\mathcal{T}_{r, n_{a}+n_{b}-2}^{2}}^{-1}(1-\alpha)$ :

$$
\begin{aligned}
T^{2}(\mathbf{T}, a, b) \leq F_{T_{r, n_{a}+n_{b}-2}^{2}}^{-1}(1-\alpha) & \Rightarrow H_{0} \text { is accepted } \\
\text { Else } & \Rightarrow H_{0} \text { is rejected }
\end{aligned}
$$

where $\alpha$ is the level of significance which can be selected to adjust the false positive and negative error rates [38]. For SDD, states $a$ and $b$ are replaced by the healthy state and an unknown current state to be classified.

The performance of a statistical hypothesis test for a certain DSF type can be evaluated using a testing dataset which contains known true positive and true negative samples with respect to the null hypothesis, i.e. samples coming from the healthy and damaged state, respectively. The accuracy, Acc, can then be calculated from the numbers of identified true positives, $n_{t p}$, and true negatives, $n_{t n}$, and the total number of samples, $n_{t o t}$, as [39]:

$$
A c c=\left(n_{t p}+n_{t n}\right) / n_{t o t}
$$

\subsection{Feature selection and objective function}

Selecting components of multivariate DSFs in a systematic way allows composing optimal DSF vectors with improved damage detection capabilities. Fast forward (FF) selection is an efficient approach for this task which has only minor computational requirements and can easily be adopted for different DSF types, i.e. initial or transformed. The FF approach consists of two steps. In the first step, a ranking of all DSF components is created iteratively beginning with a screening of candidates by evaluating a predefined objective function. The candidate with the highest objective function value is retained and receives rank one. Then, all possible bivariate DSFs are created by adding in turn the remaining components to the rank-one component, and the best of these pairs is retained. Then all possible three-tuples are examined, etc., and the process continues until all the available DSF components have been ranked. In the second step, the optimal DSF n-tuple or subset is selected which gives the overall highest objective function value.
A natural choice to be used in an SDD objective function in the context of statistical hypothesis testing is the $T^{2}$ statistic defined in Eq. (8). However, the number of DOFs of the Hotelling's probability distribution function is affected by the size of the DSF vector, thus vectors of different dimensionality cannot be directly compared. Therefore, the following relative statistical distance, $T_{r e l}^{2}(m, a, b)$, is introduced:

$$
T_{r e l}^{2}(\mathbf{T}, a, b)=T^{2}(\mathbf{T}, a, b) / F_{T_{r, n_{a}+n_{b}-2}^{2}}^{-1}(1-\alpha)
$$

where division by the inverse cumulative distribution function value $F_{T_{r, n_{a}+n_{b}-2}^{2}}^{-1}(1-\alpha)$ at a selected level of significance, $\alpha$, introduces the appropriate adjustment. Values of $T_{r e l}^{2}(\mathbf{T}, a, b) \geq 1$ indicate damage occurrence.

SDD is, however, often a multi-class problem because more than one damage state can be relevant for the assessment of a structure. Thus, the SDD objective function is defined herein as:

$$
J(\mathbf{T})=\min \left\{T_{r e l}^{2}(\mathbf{T}, h, i)\right\} \forall i \in\left\{1,2, \ldots, n_{d}\right\}
$$

where the minimum is taken between the healthy and $n_{d}$ damage states. This objective function maximises (see Eq. (6)) the separation between the damage state closest to the healthy state and thereby ensures that all the damage states are separated from the healthy one as much as possible. It can be employed for FF feature selection as well as for finding the optimal DSF projections using SPP.

\section{Illustrative bivariate example}

This section illustrates the concepts behind, and advantages of, the proposed SPP technique over using the initial or PCA-transformed DSF selections for SDD with the help of a generic, bivariate DSF. The discussions are visualized in Figure 1. Three multivariate Gaussian DSF distributions, with different means and variance-covariance matrices assumed to be known exactly, are considered to represent the healthy state and two damage states, i.e. Damage A and B, respectively. An elliptical acceptance region of the null hypothesis (Eq. (7)) is plotted for the healthy state with a thick solid line for using jointly the two initial DSFs, $v_{1}$ and $v_{2}$, or the two corresponding PCA scores. (To preserve sense of scale, it was assumed that the initial DSFs both follow a normalized zero-mean Gaussian distribution with a correlation coefficient of 0.5 , and testing is done at a $5 \%$ significance level of the $\mathcal{X}_{2}^{2}$ (chi-square with two DOFs) probability distribution applicable when the means and variance-covariance are known exactly.) If either univariate DSF $v_{1}$ or $v_{2}$ is used alone, the acceptance region is either a horizontal or vertical band (dashed lines), respectively, determined by the 


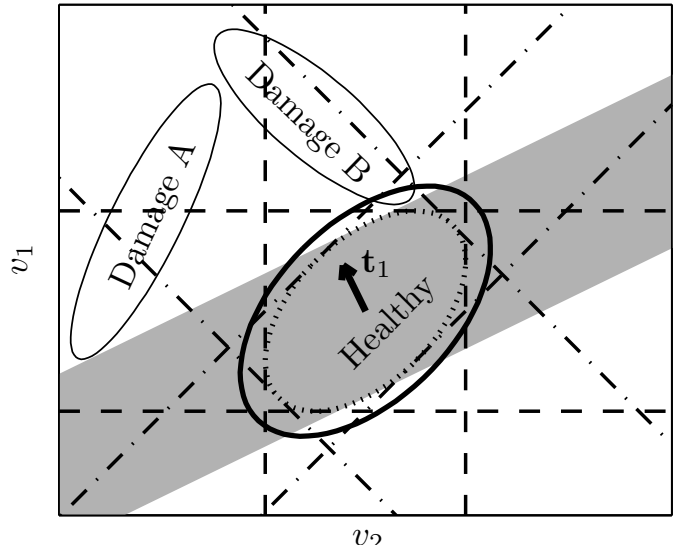

_ Bivariate initial / PCA scores

- - Univariate initial Univariate SPP

. - Univariate PCA scores ….... SPP*

Figure 1. Damage detectability for various DSF selections.

$\mathcal{X}_{1}^{2}$ (chi-square with one DOF) distribution (or more simply a normalised univariate Gaussian distribution). Similarly, projecting the initial DSFs onto single PCs results in acceptance bands (chain lines) parallel to either the major or minor axis of the bivariate ellipse.

Any of the above selections of DSFs will perform suboptimally for SDD for the considered SDD task involving Damage A and Damage B. As can be seen in Figure 1, some DSFs of Damage B may fall into the bivariate healthy state acceptance ellipse. Using only $v_{1}$, does not allow to detect Damage $\mathrm{A}$, and using only $v_{2}$ Damage $\mathrm{B}$, respectively. Any of the two univariate $\mathrm{PC}$ scores will not detect damage $\mathrm{A}$ and/or B confidently, either.

However, there are clearly univariate DSF projections, one of them indicated in Figure 1 by its projection vector $\mathbf{t}_{1}$ and the corresponding shadowed acceptance band, that allow confident detection of both Damage A and B. Further optimisation with respect to an objective function like the one in Eq. (13) can determine the optimal direction for SPP. This demonstrates the improved performance of SPP over the remaining discussed bivariate or univariate DSFs, the latter being in fact special, suboptimal cases of SPP.

Figure 1 also shows a smaller region inside the bivariate ellipse, designated as $\mathrm{SPP}^{*}$ and drawn with a dotted line. This region is the envelope of all SPP bands, i.e. its boundary is tangent to all such bands, as the projection vector $\mathbf{t}_{1}$ rotates $360^{\circ}$ around the origin. It represents the region where damage cannot be detected by any choice of the SPP vector at a selected significance level. Using the analytical geometry theory for envelopes [40], it can be shown that SPP* is in fact a scaled down version of the bivariate ellipse, where the scaling factor is equal to square root of the ratio of the inverse cumulative distribution functions $\mathcal{X}_{1}^{2}$ and $\mathcal{X}_{2}^{2}$ values at the selected level of significance.

\section{Experimental application}

Laboratory experiments with a small-scale WTB were conducted in order to demonstrate and verify the proposed methodology. This section presents first the experimental setup and the specimen. Second, the extraction of initial and optimized DSFs from acceleration response signals is discussed. Finally, the SDD method performance is assessed by evaluating statistical hypothesis testing results for previously unseen datasets.

\subsection{Wind turbine blade experiments}

The experimental configuration is shown in Figure 2. The structure under study is a $2.36 \mathrm{~m}$ long WTB of a small WT with $5 \mathrm{~kW}$ rated power output and a $5 \mathrm{~m}$ rotor diameter. The WTB was mounted in an upright position and its root fixed to a massive steel base sitting on a concrete floor. Non-contact wind-like excitations were created with the help of a domestic pedestal fan with a $40.6 \mathrm{~cm}$ diameter rotor at a zero degrees angle of attack. The WTB has a solid aerofoil E387 cross-section with a constant width of $150 \mathrm{~mm}$, as shown in Figure 3. The blade is made of a pultruded glass-fibre reinforced epoxy composite. The mass density of $2.30 \mathrm{~g} / \mathrm{cm}^{3}$ was estimated from the measured total mass of $7,110 \mathrm{~g}$. An earlier study [28] found, via experimental modal analysis, the first four WTB flap-wise modal frequencies to be $1.73 \mathrm{~Hz}$, $11.1 \mathrm{~Hz}, 31.3 \mathrm{~Hz}$ and $61.3 \mathrm{~Hz}$, respectively, and the first torsional frequency to be $38.8 \mathrm{~Hz}$.

Acceleration responses were measured using a miniature Metra KS94B-100 piezoelectric accelerometer with a sensitivity of $100 \mathrm{mV} / \mathrm{g}$ and frequency range between $0.5 \mathrm{~Hz}$ to $28 \mathrm{kHz}$. The sensor was attached to the WTB tip with adhesive wax, as indicated in Figure 2. This location was selected because it does not correspond to any node of a cantilever, thus responses contained contributions of all the modes that were excited. The acceleration signals were digitized using a National Instruments NI-9423 data acquisition card connected to a NI cDAQ-9174 chassis and a laptop. The NI LabView software was employed for signal processing.

Figure 2 shows additionally the three damage locations selected for demonstrating the proposed SDD concepts. To study different damage scenarios consisting of distinct combinations of damage locations and extents without permanently altering the specimen, non-destructive modifications were introduced by attaching small masses of between $10 \mathrm{~g}$ and $100 \mathrm{~g}$ in increments of $10 \mathrm{~g}$ to the WTB. Note even the largest 


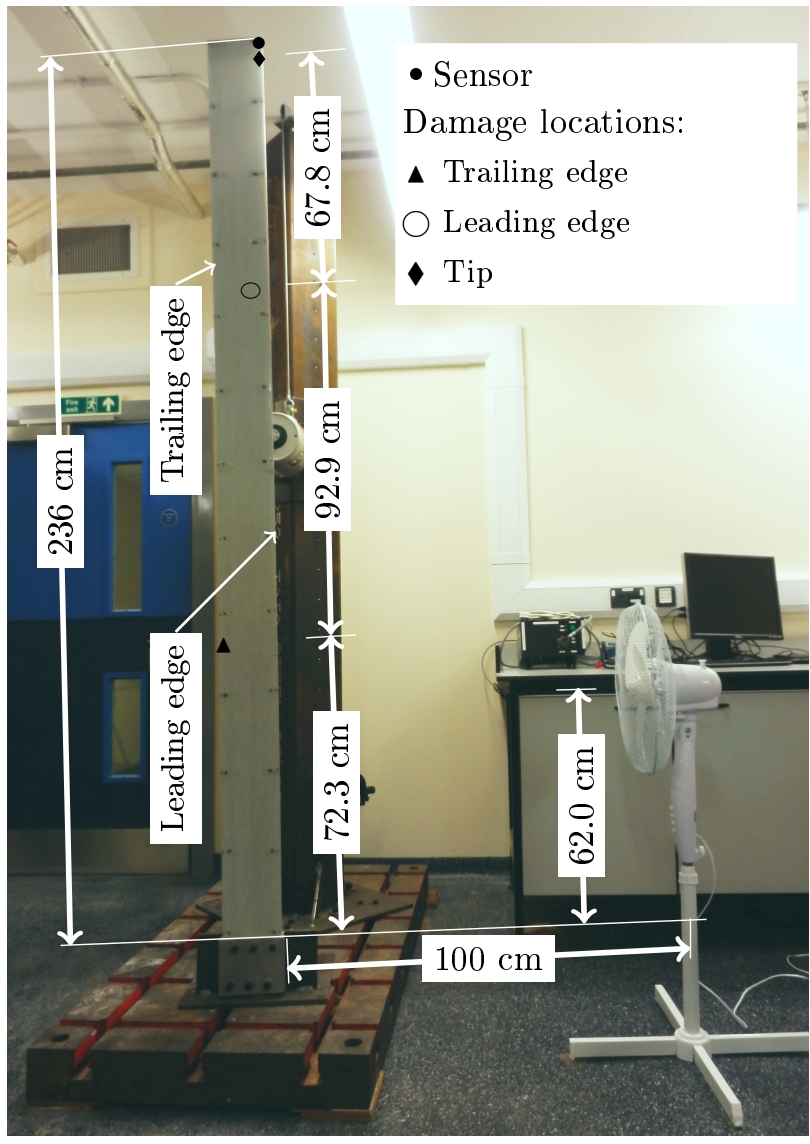

Figure 2. Experimental setup.

Trailing edge Low pressure side Leading edge

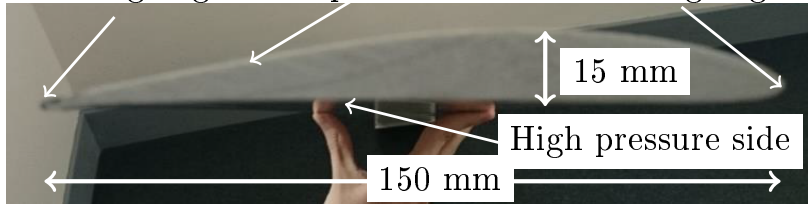

Figure 3. Cross-section of experimental wind turbine blade.

mass corresponded only to $1.4 \%$ of the total WTB mass. The locations were selected to mimic those found in full-scale, in-situ damage studies and inspection reports [41-43], where the trailing edge at $33 \%$ of the blade length from the root, the leading edge at $66 \%$ of the blade length from the root and the WTB tip were identified as damage 'hotspots'. The studied damage scenarios are summarized in Table 1, separately for training and testing cases.

\subsection{Initial and optimized damage sensitive features}

This section explains the training phase of the proposed SDD method. For each structural state, acceleration responses were acquired for 30 mins at a constant sampling rate of $2,048 \mathrm{~Hz}$. The signals
Table 1. Damage scenarios

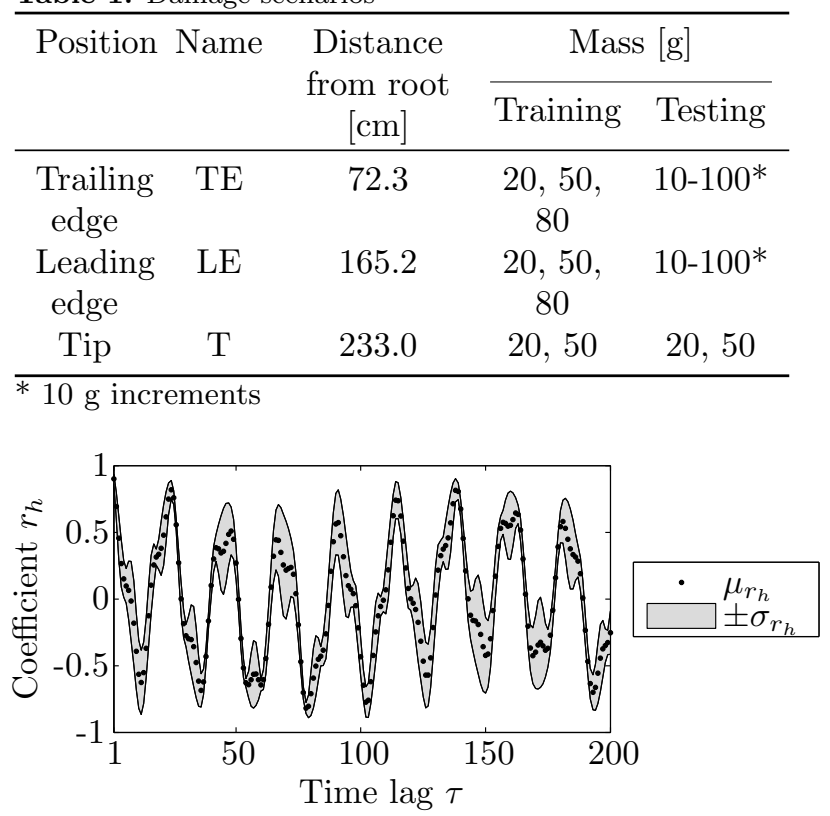

Figure 4. Estimated mean and standard deviation of ACCs in healthy state.

were pre-processed for reducing high frequency noise by filtering with an eighth order Chebyshev type I low-pass filter with a $204.8 \mathrm{~Hz}$ cut-off frequency and consecutive resampling at $256 \mathrm{~Hz}$ to observe the WTB modes that were excited by the fan. Next, each record was divided into 400 segments of a $5 \mathrm{~s}$ length and approximately $10 \%$ overlap. In order to reduce the variability between time series segments due to fluctuations of the excitation, the segments were normalized by removing the estimated means and dividing by the estimated standard deviations.

For the definition of initial DSFs, ACCs were calculated up to lag 200 from 400 segments of the healthy state data. The estimated ACC means and standard deviations are shown in Figure 4. A general periodic pattern without noticeable decay can be observed. The initial DSFs were constructed from each time series segment using ACCs from lags one to 100. This still enabled fully observing responses of the higher modes, which are normally more sensitive to localised damage, but reduced the computational demands for DSF processing. Finally, the ACCs were transformed using Fisher's $z$-transformation (Eq. (2)) so that the DSF vectors follow multivariate Gaussian distributions.

The optimization of DSFs was performed using 1,200 samples from the healthy state and 400 samples from each of the eight damage states denoted as 'training' in Table 1. SPP was performed to minimize to the SDD objective function $J(\mathbf{T})$ (Eq. (13)). The 


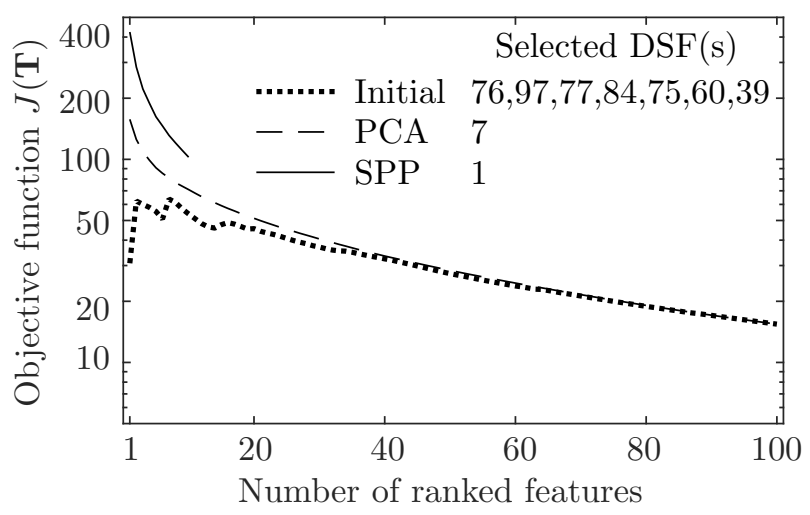

Figure 5. SDD objective function for ranked subsets of initial, PCA and SPP-transformed features (vertical axis in log scale).

sequential projection vectors were optimized by the covariance matrix adaptation-evolutionary strategy using the MATLAB ${ }^{\circledR}$ source code provided by Hansen [44]. Projection dimensions between one and 10 were studied because it was expected that a small number of scores will be sufficient for good SDD results (and this was indeed confirmed as can be seen later). Additionally, PCA was performed using the variance-covariance matrix estimated from the healthy DSF training samples only. Scores were computed by applying the SPP and PCA projection matrices to the training DSF samples from healthy and damage states.

Then, the FF approach was applied to the original and transformed DSFs in order to identify the optimal numbers of DSFs. This was done with respect to the SDD objective function, $J(\mathbf{T})$ (Eq. (13)). The results are shown in Figure 5, where the DSF subsets with the highest objective function values are additionally reported. For original DSFs, the objective function first increases until the global maximum at eight ranked features and then decays towards the overall minimum when using all available ACCs. It can be seen that the selection of the optimal DSF subset is in this case made of ACCs for lags between 39 and 97 , but no obvious pattern emerges. In contrast, the objective functions for the SPP and PCA scores attain their respective maxima for a single DSF component, the fist and seventh, respectively, and adding any more scores reduces the damage detectability. The overall best results are achieved for the first SPP score followed by the seventh PCA score. The optimal ACC subset, comprising eight initial DSFs, leads to the lowest performance. Of note is also the fact that, except for SPP, the optimal subsets do not include lower order DSFs, which are sometimes taken for SDD without carefully considering their real contribution and potential for the task.

\subsection{Structural damage detection performance}

This section investigates the performance of the proposed technique for SDD, i.e. it is the testing phase that simulates what would happen in the real operational stage. The subsets of initial, PCA and SPP DSFs identified in training and obtained from previously unseen 1,200 healthy samples as well as from 400 samples of each damage scenarios used and unused in training (see Table 1) were utilised. Statistical hypothesis testing was performed by calculating the $T_{\text {rel }}^{2}$ statistics defined in Eq. (12), where the healthy state training dataset was used for estimating the mean vector and variance-covariance matrix of the healthy reference state. Although, the $T_{\text {rel }}^{2}$ statistic allows using arbitrary numbers of samples for estimating the current state statical characteristics, employing small sample sizes can be beneficial in practical applications because decisions can be made earlier when only limited samples are available. Therefore, in the present case single DSFs vectors drawn from all the available samples in the current state, i.e. 1,200 or 400 for the unseen healthy state or each damage scenario dataset, respectively, were used. It should be born in mind, however, there is a trade-off between the sample size and the damage detectability and higher numbers of samples can reduce uncertainties of the estimates and further improve the performance.

The results of this challenging test program are shown in Figure 6, where $T_{\text {rel }}^{2}$ statistics were computed for a selected 5\% level of significance. The figure combines results for different damage locations (see axis caption at the top), extents of added mass (axis caption at the bottom) and DSFs used (axis caption on the left). By comparing the distributions of the calculated statistics visually, it can be seen that the initial DSFs show the smallest variations, the SPP scores have a larger variation with a heavier tail for higher $T_{r e l}^{2}$ statistic values, and the PCA scores show the largest variations. For the unseen healthy state data, the majority of samples fall below the statistical damage detection threshold of one marked by bold horizontal lines, thus the system is correctly identified as healthy. The detection of damage shows a common pattern for the considered DSFs, where $T_{\text {rel }}^{2}$ statistic values generally increase with increasing damage extents, the trend only appearing to be reversed beyond $80 \mathrm{~g}$ of mass added to the leading edge. Additionally, a comparison of identical damage extents but at different locations shows that damages at the tip can be more easily detected than trailing edge damages. The most difficult damage scenarios to detect are for masses attached at the leading edge.

Even though the general behaviour of the DSFs is similar, differences in the performance can be identified. The initial features led to the lowest $T_{r e l}^{2}$ 


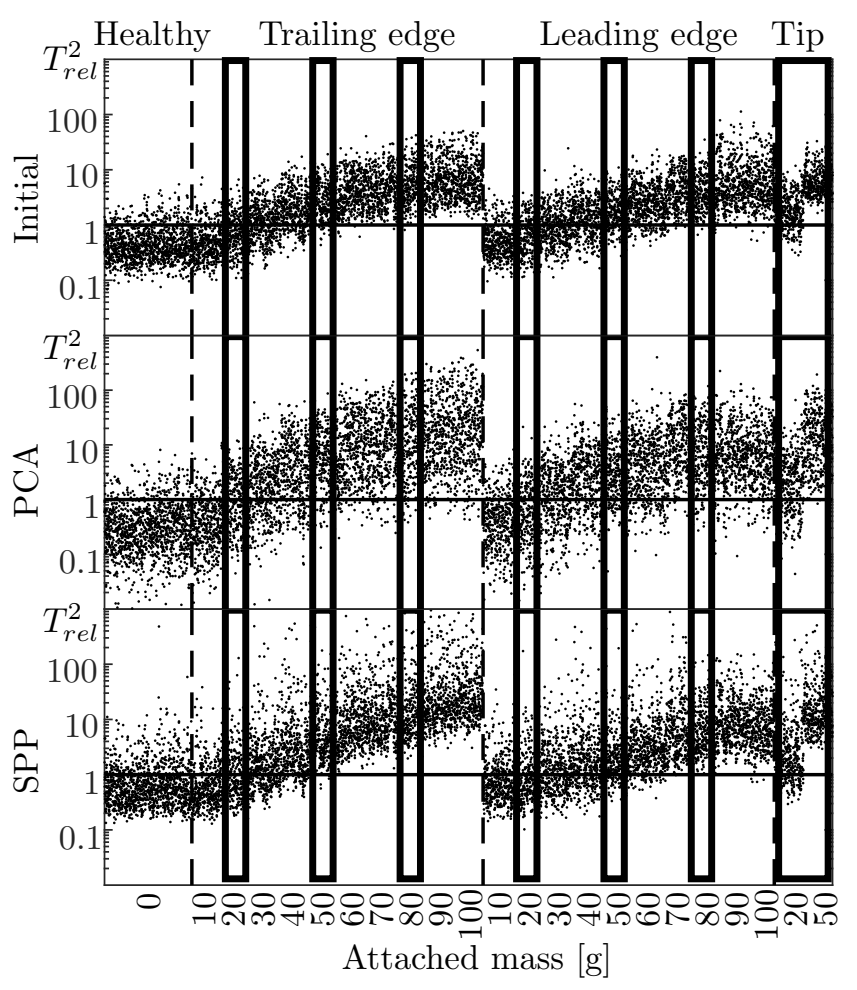

Figure 6. SDD for selected initial, PCA and SPP-transformed features (bold frames are training data; vertical axis in log scale).

statistic values, thus damages with less than $60 \mathrm{~g}$ at the trailing edge, $80 \mathrm{~g}$ at the leading edge and $50 \mathrm{~g}$ at the tip, respectively, could go undetected relatively frequently. The results for the PCA-transformed DSFs were similar to the initial features, albeit, as mentioned before, they exhibited higher variations. The SPPbased DSFs led to the best results. Here, the trailing edge damages with more than $40 \mathrm{~g}$ and leading edge damages with more than $50 \mathrm{~g}$ could be detected reliably. The tip damage of $20 \mathrm{~g}$ still proved to be a challenge to detect via SPP, but the $T_{r e l}^{2}$ statistic values were nevertheless higher than for the other two DSFs. Additionally, the accuracies defined in Eq. (11) were computed for quantitative performance assessment. This quantity incorporates all the correct healthy or damage state indications for all the samples used in Figure 6. The selected ACCs achieved an accuracy of $77.0 \%$ and the PCA scores of $77.5 \%$, respectively. The best accuracy of $79.2 \%$ was obtained for the SPPtransformed DSFs. For reference, the accuracy was also calculated using the full set of 100 ACCs with a result of only $69.3 \%$. This confirms the advantages of the overall methodology for improving the performance of DSFs by means of their optimal projections and selections.

\section{Conclusions}

The paper investigated the effects of transforming/projecting and selecting DSFs for improving the damage detectability based on statistical hypothesis testing. The DSFs were extracted from acceleration response signals of a single sensor mounted on a smallscale WTB as ACCs. A pedestal fan was employed to generate wind-like excitations, while damages were simulated non-destructively by attaching small masses of variable magnitude at three selected locations. SPP projection vectors were optimized with respect to a multivariate statistical distance between DSFs from different structural states. Additionally, PCA was performed, and optimal subsets of the initial, PCA and SPP-transformed features were identified by the FF procedure utilizing the same distance measure.

It has been shown that using a single optimized SPP projection of ACCs improves the damage detectability more than selecting components from the original or PCA-transformed features for all considered damage locations. The overall measure of SDD accuracy combining true positive and negative ratios was $79.2 \%$ for SPP, $77.5 \%$ for PCA and $77.0 \%$ for the initial features, respectively. Furthermore, SPPprojected DSFs enabled detection of smaller masses than the remaining two approaches. Future research will explore the use of different types of initial DSFs as well as the use of different selection of training damage scenarios on the method performance. An extension of the proposed methodology to damage localization and severity estimation should also be explored.

\section{Acknowledgments}

S. Hoell's work at Lloyd's Register Foundation Centre for Safety and Reliability Engineering at University of Aberdeen was supported by Lloyd's Register Foundation. The Foundation helps to protect life and property by supporting engineering-related education, public engagement and the application of research.

\section{References}

[1] Blanco M I 2009 Renewable and Sustainable Energy Reviews 13 1372-1382

[2] Farrar C R and Worden K 2007 Philosophical Transactions of the Royal Society A: Mathematical, Physical and Engineering Sciences 365 303315

[3] Li D, Ho S C M, Song G, Ren L and Li H 2015 Smart Materials and Structures 24 033001-01033001-24

[4] Kinet D, Mégret P, Goossen K, Qiu L, Heider D and Caucheteur C 2014 Sensors 14 7394-7419 
[5] Mutlib N, Baharom S, El-Shafie A and Nuawi M 2016 Structural Control and Health Monitoring 23 409-422

[6] Carden E P and Fanning P 2004 Structural Health Monitoring 3 355-377

[7] de Lautour O R and Omenzetter P 2010 Structural Control and Health Monitoring 17 614-631

[8] Hoell S and Omenzetter P 2016 Mechanical Systems and Signal Processing $\mathbf{7 0 7 1} 557-577$

[9] Nair K K and Kiremidjian A S 2007 Journal of Dynamic Systems, Measurement and Control, Transactions of the ASME 129 285-293

[10] Sohn H and Farrar C R 2001 Smart Materials and Structures 10 446-451

[11] Beskhyroun S, Oshima T, Mikami S and Miyamori Y 2013 Journal of Civil Structural Health Monitoring 3 207-223

[12] Papatheou E, Manson G, Barthorpe R J and Worden K 2010 Journal of Sound and Vibration 329 2349-2366

[13] Carbajo E S, Carbajo R S, Mc Goldrick C and Basu B 2014 Mechanical Systems and Signal Processing 47 78-93

[14] Yang Z, Yu Z and Sun H 2007 Mechanical Systems and Signal Processing 21 2918-2932

[15] Trendafilova I, Palazzetti R and Zucchelli A 2015 European Journal of Mechanics, A/Solids 49 197204

[16] Yang Z C, Wang L, Wang H, Ding Y and Dang X 2009 Journal of Sound and Vibration 325 755-768

[17] Zhang M and Schmidt R 2014 Journal of Sound and Vibration 333 7352-7363

[18] Lu K C, Loh C H, Yang Y S, Lynch J P and Law K H 2008 Smart Structures and Systems 4 759777

[19] Zimek A, Schubert E and Kriegel H P 2012 Statistical Analysis and Data Mining 5 363-387

[20] Park C, Tang J and Ding Y 2010 Structural Health Monitoring 9 59-74

[21] Zugasti E, Mujica L E, Anduaga J and Martinez F 2013 Key Engineering Materials 569-570 620627

[22] Worden K, Manson G, Hilson G and Pierce S G 2008 Journal of Sound and Vibration 309 529-544

[23] Jolliffe I T 2002 Principal Component Analysis 2nd ed (New York, USA: Springer-Verlag)

[24] Kullaa J 2003 Mechanical Systems and Signal Processing 17 163-170

[25] Mohd Aris K D, Mustapha F, Salit M S and Abdul Majid D L A 2014 Journal of Intelligent Material Systems and Structures 25 575-584
[26] Bandara R P, Chan T H T and Thambiratnam D P 2014 Engineering Structures 66 116-128

[27] Cross E J, Manson G, Worden K and Pierce S G 2012 Proceedings of the Royal Society A: Mathematical, Physical and Engineering Sciences 468 4098-4122

[28] Hoell S and Omenzetter P 2016 Structural Health Monitoring 15 685-705

[29] Guo Q, Wu W, Questier F, Massart D L, Boucon $\mathrm{C}$ and De Jong S 2000 Analytical Chemistry $\mathbf{7 2}$ 2846-2855

[30] Bendat J S and Piersol A G 2010 Random Data Analysis and Measurement Procedures 4th ed (Hoboken, USA: Wiley)

[31] Hoell S and Omenzetter P 2015 Damage detection in wind turbine blades based on time series correlations Proceedings of the 7th International Conference on Structural Health Monitoring of Intelligent Infrastructure (Torino, Italy) pp 1-10

[32] Rasch D, Pilz J, Verdooren R and Gebhardt A 2011 Optimal Experimental Design with $R$ (Boca Raton, USA: Taylor \& Francis)

[33] Friedman J H and Tukey J W 1974 IEEE Transactions on Computers C-23 881-890

[34] Espezua S, Villanueva E and Maciel C D 2014 Neurocomputing 123 40-48

[35] Bäck T, Foussette C and Krause P 2013 Contemporary Evolution Strategies Natural Computing (Heidelberg, Germany: Springer)

[36] Hansen N and Ostermeier A 2001 Evolutionary Computation 9 159-195

[37] Rencher A C 1998 Multivariate Statistical Inference and Applications (New York, USA: Wiley)

[38] Montgomery D C 1997 Introduction to Statistical Quality Control 3rd ed (Hoboken, USA: Wiley)

[39] Dougherty G 2013 Patter Recognition and Classification (New York, USA: Springer)

[40] Bruce J W and Giblin P J 1984 Curves and Singularities 1st ed (Cambridge, UK: Cambridge University Press)

[41] Ataya S and Ahmed M M Z 2013 Engineering Failure Analysis 35 480-488

[42] Jensen F M, Falzon B G, Ankersen J and Stang H 2006 Composite Structures 76 52-61

[43] Ciang C C, Lee J R and Bang H J 2008 Measurement Science and Technology 19 1-20

[44] Hansen N $2012 \quad$ cmaes.m https://www.lri.fr/ hansen/cmaes.m, Accessed: 04-August-2016 